\title{
Non-enzymatic Determination of L-Proline Amino Acid in Unprocessed Human Plasma Sample Using Hybrid of Graphene Quantum Dots Decorated with Gold Nanoparticles and Poly Cysteine: A Novel Signal Amplification Strategy
}

\author{
M. HaSanzadeh, ${ }^{* \dagger}$ H. NavaY Baghban, $* *$ and N. ShadJOU $* * *$ \\ *Drug Applied Research Center, Tabriz University of Medical Sciences, Tabriz, Iran \\ **Pharmaceutical Analysis Research Center and Faculty of Pharmacy, Tabriz University of Medical Sciences, \\ Tabriz, Iran \\ ***Department of Nano Technology, Faculty of Science, Urmia University, Urmia 57154, Iran
}

\begin{abstract}
An innovative electrochemical interface for quantitation of L-proline (L-Pro) based on ternary amplification strategy was fabricated. In this work, gold nanoparticles prepared by soft template methodology were immobilized onto green and biocompatible nanocomposite containing poly as a conductive matrix and graphene quantum dots as the amplification element. Therefore, a novel multilayer film based on poly-L-cysteine, graphene quantum dots (GQDs), and gold nanoparticles (GNPs) was exploited to develop a highly sensitive electrochemical sensor for the detection of L-Pro. Fully electrochemical methodology was used to prepare a new transducer on a glassy carbon electrode, which provided a high surface area towards sensitive detection of L-Pro. The prepared electrode was employed for the detection of L-Pro. Under optimized conditions, the calibration curve for L-Pro concentration was linear in $0.5 \mathrm{nM}-10 \mathrm{mM}$ with a low limit of quantification of $0.1 \mathrm{nM}$. The practical analytical utility of the modified electrode was illustrated by determination of L-Pro in unprocessed human plasma samples.
\end{abstract}

Keywords Amino acid, electrochemistry, graphene quantum dot, gold nanoparticle, sensor

(Received July 25, 2017; Accepted November 9, 2017; Published March 10, 2018)

\section{Introduction}

Recently, graphene quantum dots (GQDs) have been explored for their fascinating electrical and optical features and employed in variety of applications, such as photovoltaics, bioimaging, fuel cells and biosensing purposes. ${ }^{1-3}$ GQDs have distinct advantages over semiconductor inorganic QDs, such as abundancy due to their carbon base, lower toxicity, high solubility in various solvents and capability of being functionalized by attaching groups at their edges. Also, because of having semi-molecular characteristics, they can be easily handled in contrast with colloidal semiconductor QDs. ${ }^{4}$ Interestingly, GQDs having the nano-scaled graphene structure exhibit both the properties of nanomaterials and desirable physical features of the other graphene-based structures, such as carboxylic acid functional groups and the $\pi-\pi$ conjugated network. ${ }^{5,6}$ The GQDs are usually in the size range of $3-20 \mathrm{~nm}$ and have proven multivalent redox behavior in different sizes of $2.2 \pm 0.3,2.6 \pm 0.2$, and $3 \pm 0.3$, which can be recorded by voltammetry techniques and utilized for electrochemical sensing approaches. ${ }^{7}$ Besides numerous reports of optical biosensors based on GQDs, electrochemical GQDs-modified biosensors

† To whom correspondence should be addressed. E-mail: hasanzadehm@tbzmed.ac.ir; mhmmd_hasanzadeh@yahoo. com have been recently reported, as it has been demonstrated that GQDs possess superior electron donor and acceptor capability compared to microsized graphene sheets along with a greater number of carboxyl groups and smaller dimensions compared to other carbon nanomaterials, such as graphene and carbon nanotubes. Anisotropic gold nanostructures exhibit varying optical and electrocatalytical behaviors as a function of their shape and size. Up to now, several studies toward synthesis of shape-controlled nanostructures via electrochemical deposition techniques have been conducted and their excellent electrocatalytic features for sensing analytes have been concluded. $^{8-11}$ The relation between size and morphology of various gold nanostructures, i.e. roughness, dominant metal facet and surface area toward reduction of oxygen and oxidation of methanol have been evaluated, which suggests the possibility of imparting higher sensitivity to electrochemical biosensors by tailoring the morphology of nanostructures. Additionally, gold nanostructure-deposited electrode surfaces provide for a huge surface area with numerous sites for biomolecule immobilization. We have attempted to develop a sensitive electrochemical direct detection method for L-Pro and investigate the synergistic effect of gold nanoparticles (GNPs) and GQDs as effective substrate and P-Cys as conductive polymer matrix.

Fabrication of nanoparticles (NPs) into a polymer structure is a new method to enhance the conductivity of a polymer matrix and is an excellent pathway for preparation of polymers with high electrical properties, which are improved by incorporation 
of NPs. ${ }^{12}$ Application of poly-amino acids modified by NPS and the use of these nanocomposites for bioanalytical purposes has been described in recent literature. ${ }^{13}$ One of the important candidates for this purpose is L-arginine (L-Arg) and L-cysteine (L-Cys). ${ }^{14,15}$ In view of the sulfhydryl functional group in the chemical structure of L-Cys, this amino acid could be an excellent candidate for electropolymerization and lead to the preparation of a conductive interface with "poly-cysteine". On the other hand, this polymeric matrix could be functionalized by NPs for better electrochemical performance.

Proline is one of the most abundant amino acids in the cell microenvironment. Together with hydroxyproline, proline accounts for $25 \%$ of collagen, a predominant protein in the extracellular matrix (ECM). As the only proteinogenic secondary amino acid, proline has special metabolic features. ${ }^{16}$ Not only does proline have its own family of enzymes, but also its catabolism initiated by proline dehydrogenase (PRODH) is metabolically linked with several other core metabolic pathways in the human body. Therefore, detection and sensitive determination of this amino acid is highly requested by clinicians. One of the best candidates for this purpose is electrochemical sensing. ${ }^{17}$ In this work, as a continuation of our previous works in the construction of electrochemical sensors for amino acids ${ }^{18-30}$ and considering the benefits of the GQDs, GNPs, and P-Cys, we used these materials for the preparation of a novel electrochemical sensor for the detection of L-Pro in real samples. Electrochemical behavior of the prepared electrode was characterized by using cyclic voltammetry $(\mathrm{CV})$, differential pulse voltammetry (DPV) and the chronoamperometry (CHA) technique. In summary, we proposed an innovative electrochemical sensor for quantitative detection of L-Pro in unprocessed human plasma samples based on PCys-GQDsGNPs as transducing materials for modifying a glassy carbon electrode (GCE). According to electrochemical properties and suitable electrical conductivity, PCys-GQDs-GNPs may enhance electrochemical signals. So, the electron transfer efficiency could be improved. Therefore, sensitivity of the sensor was improved using the proposed modifier. The proposed sensor showed excellent sensitivity as well as long-term stability. The prepared sensor was employed for the detection of L-Pro with a linear concentration range of $0.5 \mathrm{nM}-10 \mathrm{mM}$ with a low limit of quantification of $0.1 \mathrm{nM}$.

\section{Experimental}

\section{Reagents and chemicals}

L-Cysteine, tetrachloroauric acid $\left(\mathrm{HAuCl}_{4}\right), \mathrm{Na}_{2} \mathrm{HPO}_{4}$, $\mathrm{NaH}_{2} \mathrm{PO}_{4}, \mathrm{NaCl}$ and other chemical materials were purchased from Sigma-Aldrich. Phosphate buffer saline (PBS) solution $(0.1 \mathrm{M})$ was prepared with $\mathrm{Na}_{2} \mathrm{HPO}_{4}(0.2 \mathrm{M})$ and $\mathrm{NaH}_{2} \mathrm{PO}_{4}$ $(0.2 \mathrm{M})$ and adjusted to $\mathrm{pH} 7.4$ using deionized water.

Human plasma samples were obtained from the Iranian Blood Transfusion Research Center (Tabriz, Iran). Aliquots of plasma were transferred into micro-tubes and frozen at $-20^{\circ} \mathrm{C}$ until analysis. Also, human plasma samples were kept in citrated tubes and stored at $4^{\circ} \mathrm{C}$. Human plasma samples were thawed at room temperature daily and were vortexed to ensure homogeneity. After thawing the samples gently, $0.5 \mathrm{~mL}$ of an aliquot volume of this sample was spiked with L-Pro. Approximately, $0.5 \mathrm{~mL}$ of plasma was taken and added to achieve a total volume of $7 \mathrm{~mL}$.

\section{Apparatus}

Electrochemical measurements were conducted using a
(A)

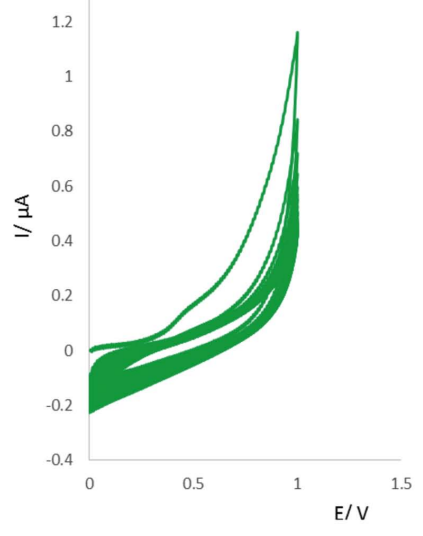

(C)
(B)

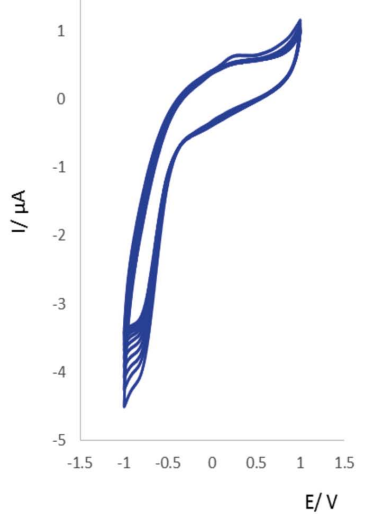

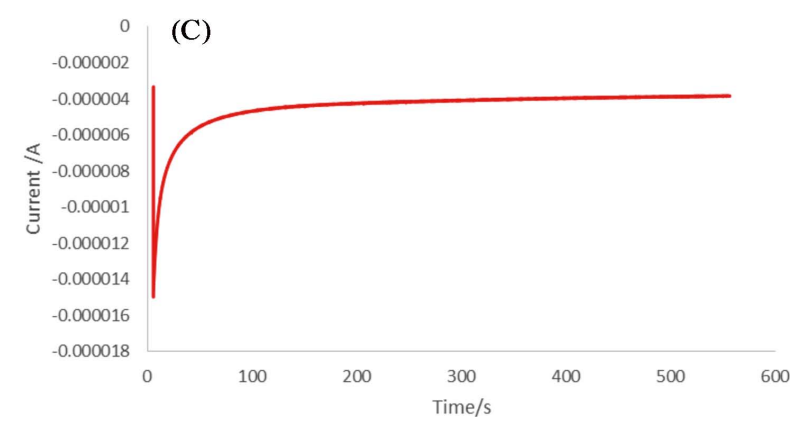

Fig. 1 A) CVs for electrodeposition of GQDs on GCE after 30 cycles in the potential range of 0 to $1 \mathrm{~V} v s$. $\mathrm{Ag} / \mathrm{AgCl}$ with $0.1 \mathrm{~V} / \mathrm{s}$ scan rate. B) Electrochemical polymerization of L-Cys on GQDs-GCE. The cyclic voltammogram depicts gradual growth of P-Cys after 10 successive cycles in the range of -1 to 1 with $0.1 \mathrm{~V} / \mathrm{s}$ scan rate. C) Chronoamperogram for the electrochemical deposition of GNPs on GCE electrode. The applied potential and the record time were $E=0 \mathrm{~V}$ vs. $\mathrm{Ag} / \mathrm{AgCl}$ and $600 \mathrm{~s}$, respectively.

conventional three-electrode cell (from Metrohm), containing an $\mathrm{Ag} / \mathrm{AgCl}$-saturated $\mathrm{KCl}$ (from Metrohm) as reference, platinum wire as counter electrodes and GCE $(d=2 \mathrm{~mm})$ as working electrode. It was powered by an electrochemical system comprised of an AUTOLAB system with PGSTAT 302N (Eco Chemie, Utrecht, The Netherlands). The system was run on a PC using NOVA 1.11 software.

The surface morphology of the electrode surface was characterized by high-resolution field emission scanning electron microscope (FE-SEM, Hitachi-SU8020, Czech) with an operating voltage of $3 \mathrm{kV}$, and chemical compositions of the GNP were analyzed by an energy dispersive spectroscopy (EDX) coupled with the FE-SEM equipment.

\section{Results and Discussion}

Electrodeposition of GQDs on the surface of P-Cys modified GCE

The graphene-based materials, such as graphene oxide, reduced-graphene oxide and graphene sheets, etc., have been widely incorporated in electrochemical (bio)sensors for designing various sensing schemes. ${ }^{31-38}$ These materials have proven applicable for purposes (electrode surface modification, signal tag, etc.) owing to their unique mechanical properties, excellent electrical conductivity and catalytic behaviors. GQD, the new member of the graphene-based materials group, combines all the unique features of these materials with nano 

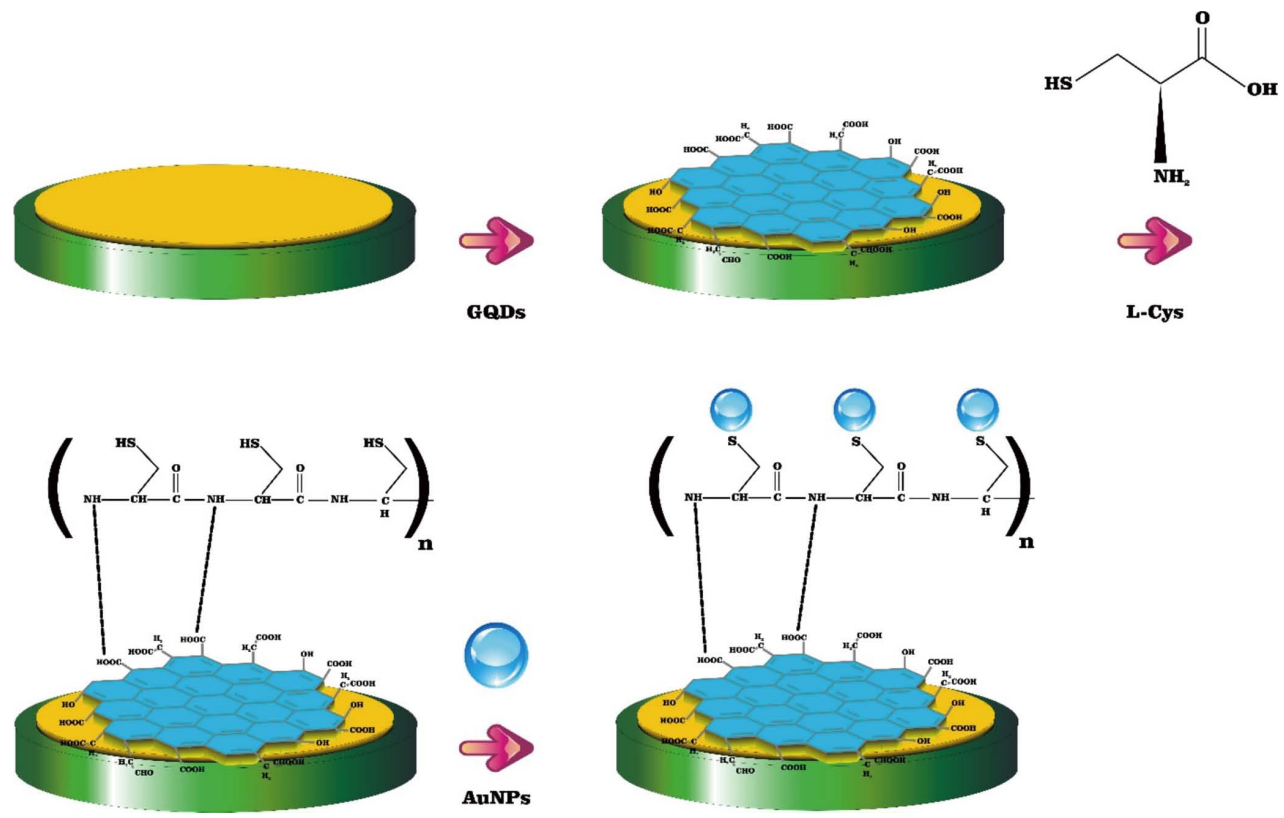

Scheme 1 Fabrication procedure of P-Cys/GQDs/GNPs-GCE.

features, which makes it highly preferential for modifying electrochemical sensing platforms. ${ }^{39-43}$

The procedure for electrodeposition of GQDs on a GCE surface was previously reported in the literature. ${ }^{39}$ Herein, the electrochemical deposition of GQDs on a GCE is considered.

In this work, the GCE was placed in the cell containing $5 \mathrm{~mL}$ of GQDs and subsequently, electrochemical deposition of GQDs on the electrode surface was conducted through $\mathrm{CV}$ with 30 consecutive cycles in the potential range of 0 to $1 \mathrm{~V} v s . \mathrm{Ag} / \mathrm{AgCl}$ (Fig. 1A).

Electropolymerization of $L-C y$ s on the surface of GQD modified GCE

Aiming to develop a uniform thin film of P-Cys on the surface of a GQD modified GCE, the electropolymerization of L-Cys was implemented on the surface of a GQD modified GCE by immersing the electrode in $0.005 \mathrm{M}$ L-Cys/PBS solution and applying $\mathrm{CV}$ in the potential range of -1 to $1 \mathrm{~V} v s$. $\mathrm{Ag} / \mathrm{AgCl}$ for 10 cycles (Fig. 1B). The cysteine-coated GQDs-GCE was rinsed with deionized water and prepared for the next modification step.

It is predicted that the hydroxyl functional groups of GQDs will interact with the free thiol group P-Cys layer, establishing highly powerful chemical bonding via electrostatic interaction, which would help stabilize the electrodeposited GQDs layer on the surface of the electrode.

Electrodeposition of GNPs on the surface of P-Cys-GQD modified GCE

Previous studies have revealed the dependency of deposition potential on the size and morphology of generated GNPs. Researchers demonstrated that the crystal density of generated GNPs increases by shifting toward negative electrodeposition potentials from 0.3 to $-0.3 \mathrm{~V}$ on an ITO electrode, because of the increase in nucleation rate when oversaturation occurs at higher overpotentials. ${ }^{44}$ The reduction of $\mathrm{AuCl}_{4}{ }^{-}$initiates at $0.15 \mathrm{~V}$, leading to full nucleation at $E=0.20 \mathrm{~V}$, therefore, the potential was adjusted on $E=0 \mathrm{~V}$ where no reaction occurs. ${ }^{44}$ For electrodeposition of gold nanoseeds on the surface of GCE pre-modified by P-Cys-GQDs, the electrodeposition of metallic gold ions by chronoamperometry technique was carried out in the cell containing a triple solution of $20 \mathrm{mM} \mathrm{HAuCl}_{4}, 500 \mathrm{mM}$ $\mathrm{H}_{2} \mathrm{SO}_{4}$ and $50 \mathrm{mM}$ arginine. The electrodeposition potential was set at $E=0 \mathrm{~V}$ and time duration of the chronoamperometry was adjusted $500 \mathrm{~s}$ (Fig. 1C). For the fabrication mechanism of GNPs, it can be mentioned that gold atoms or clusters are initially and rapidly formed, followed by promoted nucleation. The arginine adsorption process causes formation of a GNPstable arginine layer in which arginine migrates to the surface via the zwitterions functional group, and its positively charged guanidinium functional group tends to move outward into the solution. Therefore, $\mathrm{AuCl}_{4}{ }^{-}$ions were electrostatically absorbed on the surface of P-Cys and caused preferential growth of Au by the diffusion-limited aggregation mechanism. Scheme 1 shows all of preparation steps.

SEM and EDX characterization of the modified sensing interface SEM imaging was applied to evaluate the morphology of the electrodeposited layers. The SEM images of the electrode surfaces related to P-Cys-GCE, P-Cys/GQDs-GCE, and P-Cys/ GQDs/GNPs-GCE are represented in Fig. 2. The SEM images reveal scattered particles of L-cysteine in a broad size range with relatively low coverage (Fig. 2A); the evenly deposited, and closely packed GQDs layer in approximate size range of $30 \mathrm{~nm}$ (Fig. 2B); and the uniform GNPs layer with particles of similar non-spherical morphology related to P-Cys/GQDs/GNPs-GCE (Fig. 2C). The EDX characterization technique was employed to further study the formation of P-Cys-GQDs and P-CysGQDs-GNPs layers on the surface of GCE (Fig. 2).

As evident in Fig. 3A, signals for $\mathrm{C}$ and $\mathrm{O}$ elements belong to both cysteine and GQDs layers, while $\mathrm{N}$ is only attributed to the $\mathrm{P}-\mathrm{Cys}$ layer, as cysteine includes amine groups. $\mathrm{Si}$ and $\mathrm{Al}$ elements are probably due to the presence of some impurities in the solid electrode. The large and sharp Au peak is the typical signal for bulk gold element. The corresponding EDX spectra of P-Cys-GQDs-GNPs-GCE (Fig. 3C) demonstrates the increase in $\mathrm{Au}$ peak, which clearly indicates the electrodeposition of nano-size gold layer. 

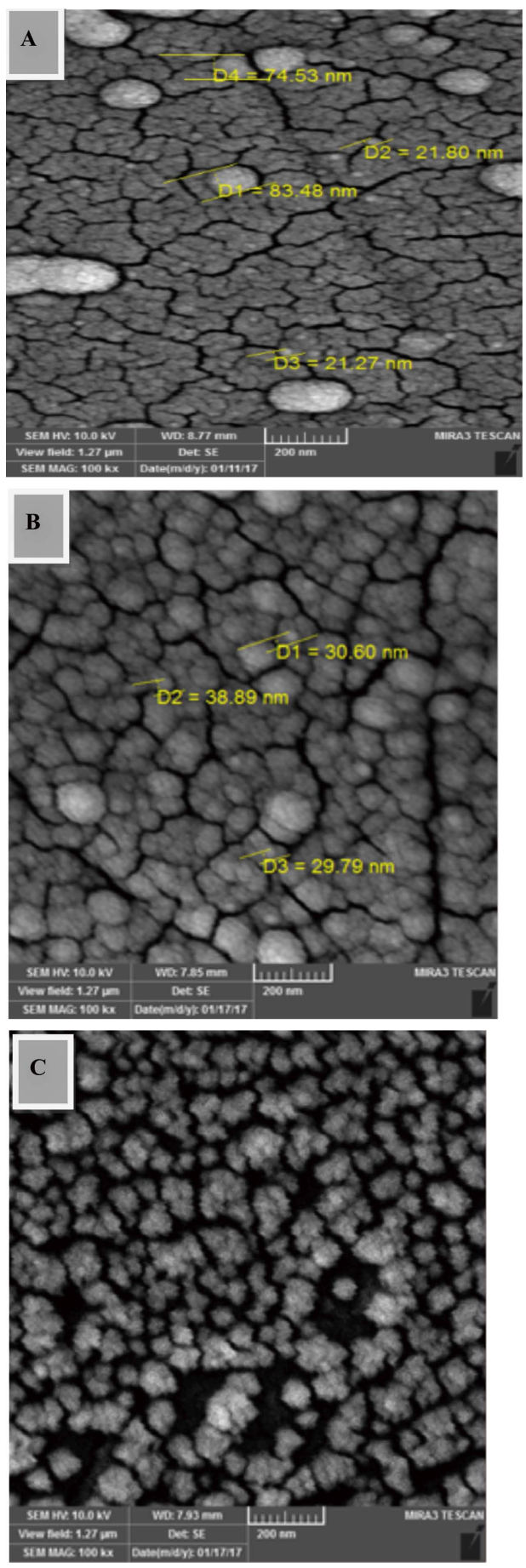

Fig. 2 FE-SEM images of the sequential modified layers on GCE: A) P-Cys-GCE, B) P-Cys/GQDs-GCE and C) P-Cys/GQDs/GNPsGCE in similar magnification.

\section{Electrochemical characterization of the sensor}

$\mathrm{CV}$ profiles in the applied potential range of -1 to $1 \mathrm{~V} v s$. $\mathrm{Ag} / \mathrm{AgCl}$ in $\mathrm{PBS}$ solution for the different modifications of GCE, namely bare GCE, the modified GCE with P-Cys, P-Cys/ GQDs and P-Cys/GQDs/GNPs are shown in Fig. 4A.

Therefore, four types of modified electrodes were evaluated for their electrochemical behavior in blank solution and the solution containing $34 \mathrm{mM}$ of L-Pro. The comparison of CVs in the absence and presence of L-Pro with different modified electrodes demonstrates a new anodic peak at $-1.62 \mathrm{~V} v s$. $\mathrm{Ag} / \mathrm{AgCl}$ on the surface of P-Cys/GQDs/GNPs-GCE. These
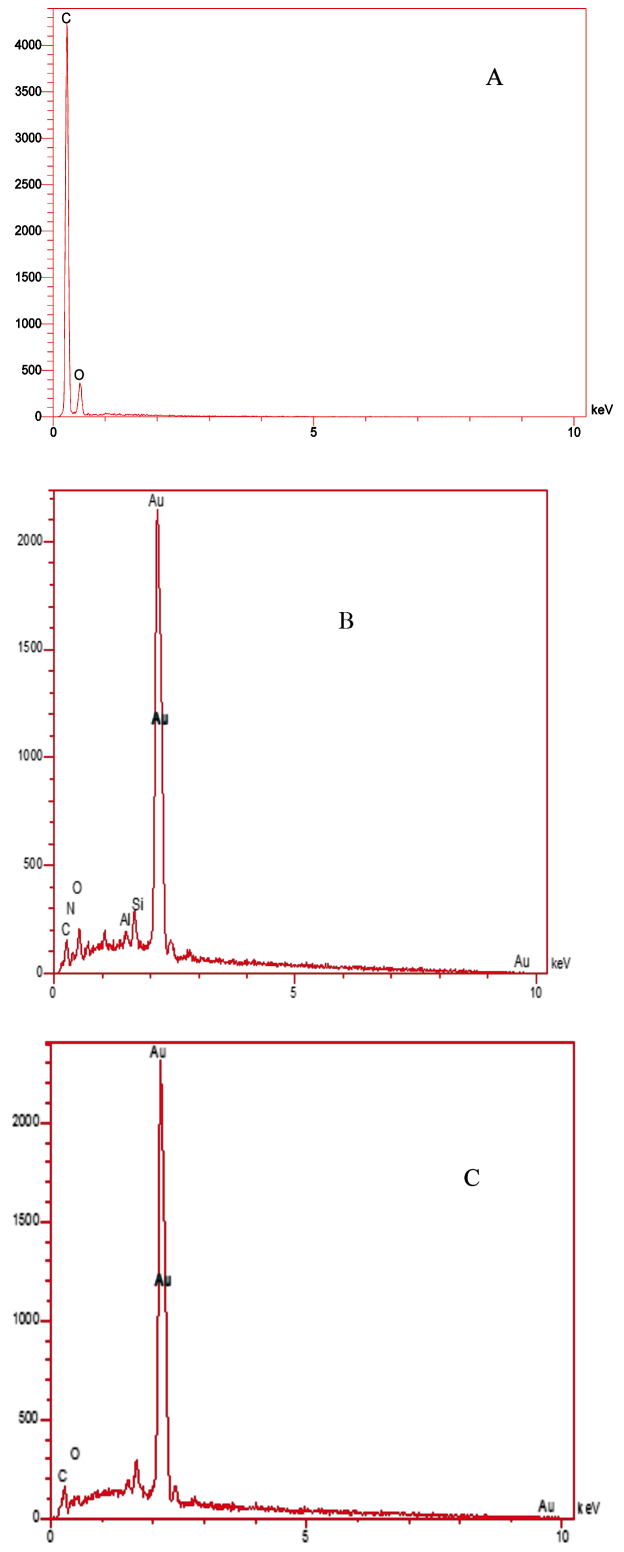

Fig. 3 EDX spectra of A) GQDs, (B) P-Cys-GQDs-GCE, and (C) P-Cys-GQDs-AuNPs-GCE.

results confirmed that the prepared nanocomposite could accelerate the rate of electron transfer that is necessary for redox reaction. Therefore, $\mathrm{P}-\mathrm{Cys} / \mathrm{GQDs} / \mathrm{GNPs}$ is a suitable mediator to shuttle electrons between L-Pro and the working electrode. This observation is also linked to the high conductivity and inherent ability of GQDs/GNPs. That might be related to the excellent properties of P-Cys as a conductive matrix and GQDs/ GNPs as dual amplification elements.

\section{Kinetic behaviors of the P-Cys/GQDs/GNPs-GCE}

Kinetic behaviors of the proposed prepared sensor were evaluated by performing $\mathrm{CV}$ indifferent scan rates from 0.02 to $0.2 \mathrm{~V} / \mathrm{s}$. As evident in the CV plot (Fig. 4B); the width of the voltammogram widened gradually and the anodic peak currents were raised considerably by an increase of scan rate. It can be concluded that at the slower scan rates, the electron exchange occurs more slowly at the electrode surface to record peak currents leading to a narrower voltammogram with shorter current peaks. 

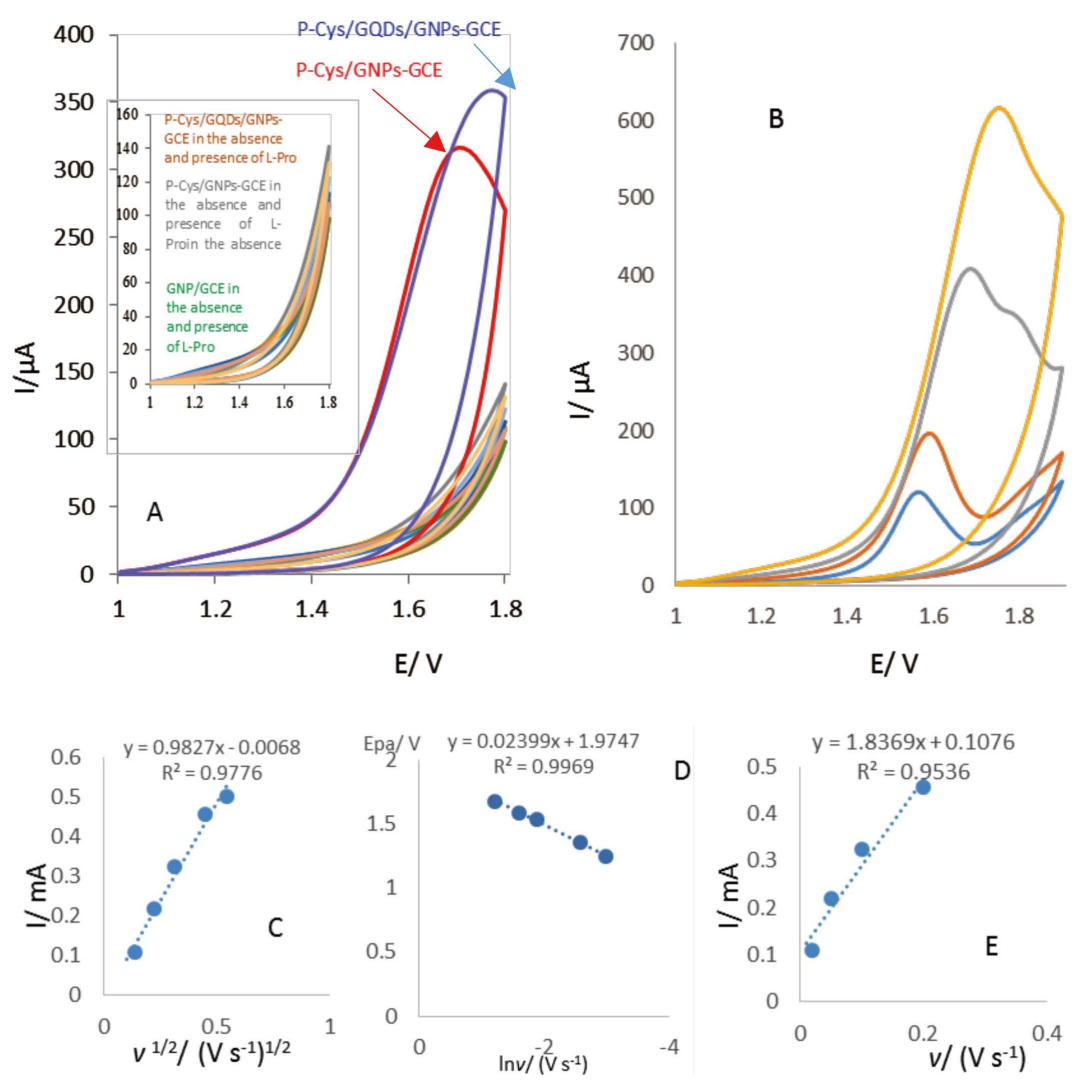

Fig. 4 A) Cyclic voltammograms for five types of electrodes: GCE, GNPs/GCE, P-Cys/GQDs/GCE, GQDs/GCE, and P-Cys/GQDs/GNPs-GCE in the absence and presence of L-Pro. B) CVs of the P-Cys/ GQDs/GNPs-GCE at different scan rates from $0.02-0.2 \mathrm{~V} / \mathrm{s}$. C) Dependence of anodic peak currents to the square root of scan rates using P-Cys/GQDs/GNPs-GCE electrode. D) Dependence of peak potential versus Napierian logaritm of sweep rates. E) The relationship between anodic peaks versus scan rates.

The scan rate analysis revealed the direct dependence of the peak current on the selected range of sweep rates. As evidenced in Figs. 4A and 4B, the slopes for the relationship of currentpotential to the anodic peak current are linear. In order to investigate the nature of the electrode reaction, dependency of the peak current versus the $\left(v^{1 / 2}\right)$ and Napierian logarithm of peak current versus the Napierian logarithm of the sweep rate (ln $v$ ) for the peaks were plotted. ${ }^{45}$ The linear dependency for $I_{\mathrm{p}}-v^{1 / 2}$ and $\ln I_{\mathrm{p}} v s$. $\ln v$ suggests the diffusion controlled nature of the three electrochemical reactions (Figs. 4C and 4D). Therefore, it can be claimed that the electrochemical processes followed the Randles-Sevcik equation, specific for diffusion controlled electrochemical reactions: ${ }^{45}$

$$
I_{\mathrm{p}}=2.69 \times 10^{5}(A D)^{(1 / 2)} n^{1.5} v^{0.5} C
$$

Here, $I_{\mathrm{p}}$ is the peak current (A), $A$ stands for the electroactive surface area $\left(\mathrm{cm}^{2}\right), D$ is to the diffusion coefficient of the molecule in solution $\left(\mathrm{cm}^{2} \mathrm{~s}^{-1}\right), n$ is the number of transferred electrons, $v$ is the scan rate $\left(\mathrm{V} \mathrm{s}^{-1}\right)$ and $C$ corresponds to the bulk concentration of the analyte $\left(\mathrm{mol} \mathrm{cm}^{-3}\right)$.

Also, the redox peak currents increased proportionally to the sweep rate, indicating that the sensor system has an electrocatalytic property of an irreversible nature. On the other hand, to elucidate the mechanism of mass transfer, two methods were used. First, the investigation of the variation of $I_{\mathrm{p}}$ versus $v^{1 / 2}$ (Fig. 4C). Using this approach, the intercept of the $I_{\mathrm{p}}$ versus $v^{1 / 2}$ was found to be $10^{-5}$, which indicates that the mass transfer was controlled by a diffusion mechanism. ${ }^{45}$ Second, investigation of the variation of $\ln I_{\mathrm{p}}$ versus $\ln v$. The results have been illustrated in Fig. 4D. The slopes of the plot in Fig. 4D for oxidation and reduction peaks were found to be 0.6133 and 0.6874 , respectively. If the slope of $\ln I_{\mathrm{p}}$ versus $\ln v$ is close to 0.5 , the process of diffusion is controlled. Thus, if the slope is close to 1 , the process is controlled through adsorption. ${ }^{45}$ Thus, the slope was calculated to be about 0.6; it can be concluded that the mass transfer is a diffusion controlled process that is in agreement with the results of the first approach.

Also, the mass transfer mechanism can also be evaluated by electron transfer coefficient, $\alpha$, of Eq. (2): ${ }^{45}$

$$
E_{\mathrm{p}}=(R T / 2 \alpha F) \ln v+\text { constant }
$$

where, $E_{\mathrm{p}}$ is electrode potential, $R$ is gas constant $\left(8.314, \mathrm{~J} \mathrm{~K}^{-1}\right.$ $\left.\mathrm{mol}^{-1}\right), T$ is temperature in Kelvin scale $(298 \mathrm{~K}), F$ is Faraday's constant $\left(96486, \mathrm{~A} \mathrm{~s} \mathrm{~mol}^{-1}\right)$, and $v$ is potential sweep rate $(\mathrm{V} / \mathrm{s})$. If the value of $\alpha$ is close to 0.5 , the system is diffusion controlled. ${ }^{45}$ Using this equation, the $\alpha$ value was obtained as 0.33 , which further supports the promise that the mass transfer of the system is diffusion controlled. In Fig. 4E, the plot of Eq. (2) obtained from $\ln I_{\mathrm{p}}$ versus $E_{\mathrm{p}}$ (Tafel plot) have been illustrated.

The special surface coverage $\left(\Gamma^{*}\right)$ was calculated via the slope of the redox peak currents (A) versus sweep rate $(\mathrm{V} / \mathrm{s})$ using Eq. (3): 


$$
I_{\mathrm{p}}=\left\{\left(n^{2} F^{2}\right) / 4 R T\right\} v A \Gamma^{*}
$$

where, $n$ is the number of transferred electrons, $F$ is Faraday's constant $\left(96486, \mathrm{~A} \mathrm{~s} \mathrm{~mol}^{-1}\right), R$ is gas constant $\left(8.314, \mathrm{~J} \mathrm{~K}^{-1}\right.$ $\left.\mathrm{mol}^{-1}\right), T$ is temperature in Kelvin scale $(298 \mathrm{~K}), v$ is potential sweep rate $(\mathrm{V} / \mathrm{s}), A$ is total electrode surface $\left(\mathrm{cm}^{2}\right)$, and $\Gamma^{*}(\mathrm{~mol}$ $\mathrm{cm}^{-2}$ ) is special surface covered with P-Cys/GQDs/GNPs-GCE. Based on the obtained results, the slope of redox peak currents versus sweep rates was 0.0002 and the $\Gamma^{*}$ value was calculated as $7.11 \times 10^{-9} \mathrm{~mol} \mathrm{~cm}^{-2}$. When P-Cys/GQDs/GNPs-GCE was used as the electrode modifier, the $\Gamma^{*}$ value was improved. Therefore, by using P-Cys/GQDs/GNPs-GCE as electrode modifier, it is possible to increase the efficiency of the prepared sensor.

\section{Chronoamperometry study}

The standard L-Pro samples in the PBS solution, as detection medium, were used to evaluate the sensitivity of the prepared L-Pro prepared sensor by employing the chronoamperometry technique. As the followed protocol was performed without incorporating redox pair and mediation, first, the potential analytical range for L-Pro sample was investigated considering the behaviors of electrodes with different modifications in -1 to $1.8 \mathrm{~V} v s . \mathrm{Ag} / \mathrm{AgCl}$ potential range. In the next stage, the analytical potential area with the least interferences in the $\mathrm{CV}$ plots was evaluated and considering the forth modification of the GCE (P-Cys/GQDs/GNPs/GCE) (Fig. 5), $1.68 \mathrm{~V} v s$. $\mathrm{Ag} / \mathrm{AgCl}$ was spotted as the desired analytical potential range.
Finally, chronoamperometry was applied in the fixed value of $1.68 \mathrm{~V}$ for tracing the antibody-antigen immunoreaction, which exhibited an orderly decline in current upon decrease in the L-Pro concentration.

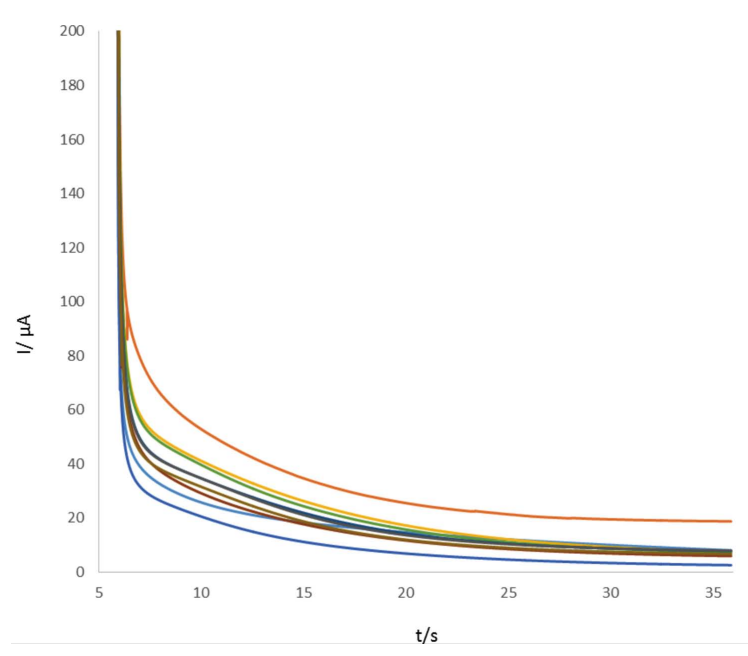

Fig. 5 Chronoamperogram for standard analysis of L-Pro in different concentrations. $E=1.29, t=50 \mathrm{~s}$ and supporting electrolyte is $0.1 \mathrm{M}$ PBS (pH 7.4).
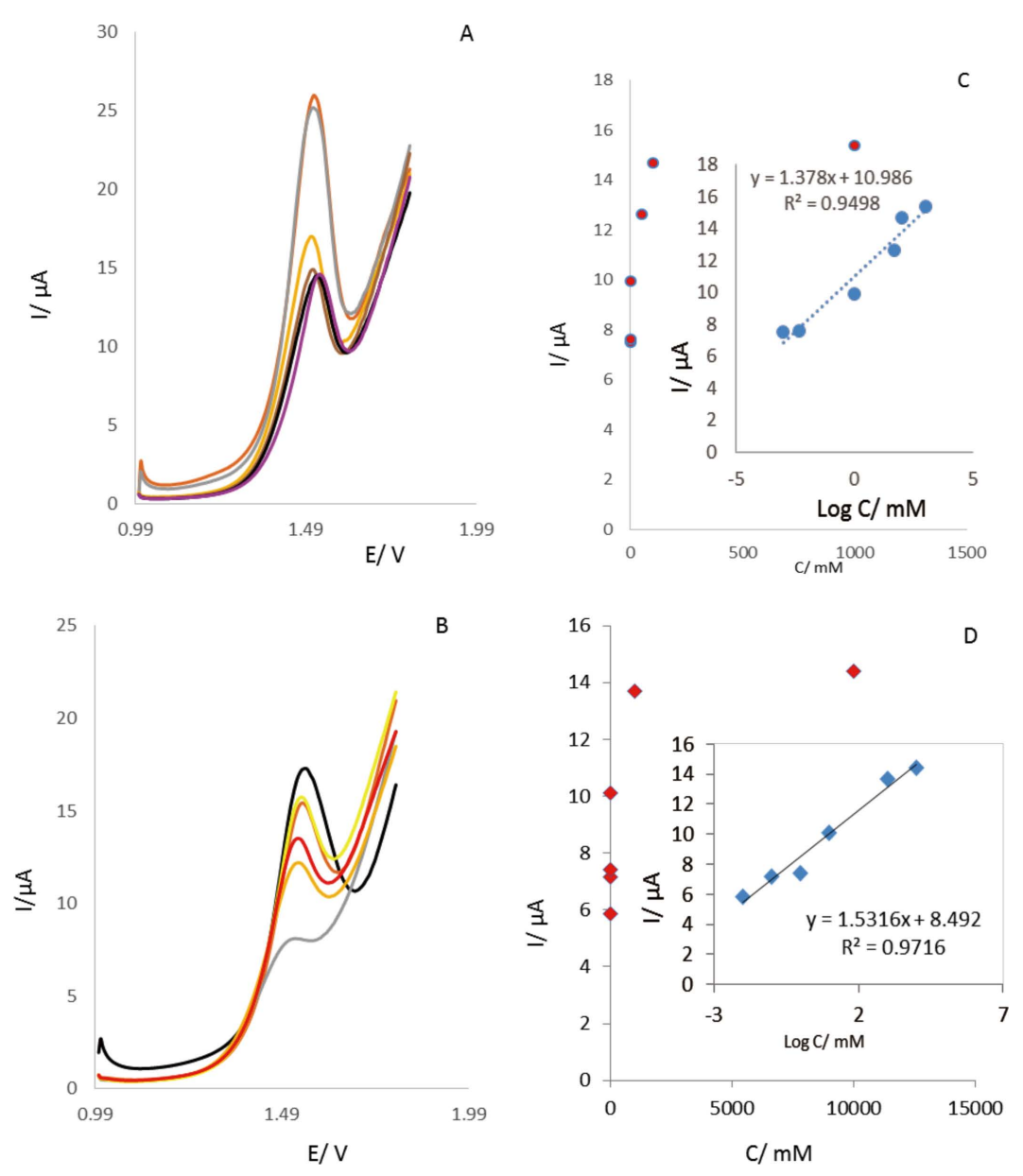

Fig. 6 DPVs of P-Cys-GQDs/GNPs-GCE in different concentrations of L-Pro in the standard (A) and human plasma samples (B). Calibration curve of L-Pro in the standard (C) and human serum samples (D). 
Table 1 Analytical parameters for determination of L-Pro in the standard and unprocessed human plasma samples obtained by DPV technique

\begin{tabular}{llrcc}
\hline \multicolumn{1}{c}{ Sample } & Slope & Y-intercept & $\begin{array}{c}\text { Range/ } \\
\mathrm{M}\end{array}$ & $\begin{array}{c}\text { LLOQ/ } \\
\mathrm{nM}\end{array}$ \\
\hline Standard solution & 1.378 & 10.986 & $0.5 \mathrm{nM}-10 \mathrm{mM}$ & 0.1 \\
$\begin{array}{l}\text { Unprocessed human } \\
\text { plasma }\end{array}$ & 1.5316 & 8.492 & $0.0001-10 \mathrm{mM}$ & 0.5 \\
\hline
\end{tabular}

\section{Analytical approach}

As a highly sensitive electrochemical method, the DPV has much higher current sensitivity and better resolution compared to CV. Therefore, the DPV method is used for determination of L-Pro in the standard and human plasma samples using P-Cys/ GQDs/GNPs-GCE. Under the optimum conditions, with the increase of L-Pro concentrations, the DPV oxidation peaks increased gradually in standard (Fig. 6A) and human plasma media (Fig. 6B). The results demonstrated that anodic peak current were proportional to one range of L-Pro concentration intervals (Figs. 6C and 6D). Table 1 revealed the analytical performance of the proposed method in human plasma samples. This range of detection and LLOQ are suitable for sensing L-Pro concentration in human plasma samples.

\section{Conclusions}

We have developed an ultrasensitive electrochemical sensing platform for L-Pro detection based on P-Cys as conductive polymeric matrix and GQDs/GNPs as a synergetic signal amplification element. For the first time, fully electrochemical methodology was used to prepare a new transducer on GCE which provided a high surface area for diffusion a high surface concentration of L-Pro. Also, GNPs were electrodeposited on the surface of P-Cys-GQDs with the assistance of amino acid as a soft template. Therefore, a green electrodeposition method was developed for the preparation of a new electrochemical interface. Experimental results show that, proposed interface can effectively improve electron transfer efficiency based on synergistic effect of P-Cys as conductive matrix and GQDs/ GNPs as dual amplification elements. It is found that, the new morphology of GNPs synthesized in the presence of soft template facilitated the electron transfer. Also, P-Cys and GQDs provided a biocompatible environment with a suitable geometric surface area for the further immobilization of GNPs. The prepared interface was employed for the detection of L-Pro in physiological $\mathrm{pH}$. Under optimized condition the calibration curve for L-Pro concentration was linear up to $0.5 \mathrm{nM}-10 \mathrm{mM}$ with a low limit of quantification of $0.1 \mathrm{nM}$. Hopefully, the immobilized strategy and the detection method can be further developed for other amino acids and biomarkers.

\section{Acknowledgements}

We gratefully acknowledge Tabriz University of Medical Sciences for financial and instrumental support for this research.

\section{References}

1. M. Hasanzadeh, A. Karimzadeh, S. Sadeghi, A.
Mokhtarzadeh, N. Shadjou, and A. Jouyban, J. Mater. Sci.: Mater. El., 2016, 27, 6488.

2. N. Shadjou, M. Hasanzadeh, F. Talebi, and A. P. Marjani, Mater. Sci. Eng. C, 2016, 76, 666.

3. M. Hasanzadeh and N. Shadjou, Mater. Sci. Eng. C, 2017, $71,1313$.

4. N. Shadjou, M. Hasanzadeh, M. Marandi, and A. Hasanzadeh, J. Mater. Sci.: Mater. El., 2016, 27, 11834.

5. X. Xu and Y. Wang, Anal. Sci., 2015, 31, 787.

6. N. Shadjou, M. Hasanzadeh, and A. Omari, Anal. Biochem., 2017, 539, 70 .

7. N. Hashemzadeh, M. Hasanzadeh, N. Shadjou, J. EivaziZiaei, M. Khoubnasabjafari, and A. Jouyban, J. Pharm. Anal., 2016, 6, 235.

8. H. Malekzad, M. Hasanzadeh, N. Shadjou, and A. Jouyban, Int. J. Biolog. Macromolecules, 2017, 105, 522.

9. M. Hasanzadeh, H. Navay baghban, A. Mokhtarzadeh, N. Shadjou, and S. Mahboob, Int. J. Biolog. Macromolecules, 2017, 105, 1337.

10. M. Hasanzadeh, H. Navay Baghban, N. Shadjou, and A. Mokhtarzadeh, Int. J. Biolog. Macromolecules, 2018, 107, 1348.

11. M. Hasanzadeh, N. Razmi, N. Shadjou, A. Mokhtarzadeh, and S. Mahboob, Int. J. Biolog. Macromolecules, 2018, 108,69

12. M. Hasanzadeh, S. Hassanpour, A. Saadati Nahr, N. Shadjou, A. Mokhtarzadeh, and S. Mahboob, Anal. Bioanal. Electrochem., 2018, 10, 128.

13. R. Shadjou, M. Hasanzadeh, M. Heidar-pour, and N. Shadjou, J. Mol. Recognit., 2018, DOI: 10.1002/jmr.2699.

14. M. Hasanzadeh, F. Mokhtari, N. Shadjou, A. Eftekhari, A. Mokhtarzadeh, V. Jouyban-Gharamaleki, and S. Mahboob, Mater. Sci. Eng. C, 2017, 75, 247.

15. J. Soleymani, M. Hasanzadeh, M. Eskandani, M. Khoubnasabjafari, N. Shadjou, and A. Jouyban, Mater. Sci. Eng. C, 2017, 77, 790.

16. J. M. Phang and W. Liu, O. Zabirnyk, Annu. Rev. Nutr., 2010, 30, 441

17. M. Hasanzadeh, A. Saadati Nahr, S. Hassanpour, N. Shadjou, A. Mokhtarzadeh, and J. Mohammadi, Enzyme Microb. Tech., 2017, 105, 64.

18. M. Hasanzadeh, G. Karim-Nezhad, N. Shadjou, M. Hajjizadeh, B. Khalilzadeh, L. Saghatforoush, M. H. Abnosi, A. Babaei, and S. Ershad, Anal. Biochem., 2009, 389, 130

19. M. Hasanzadeh, N. Shadjou, S.-T. Chen, and P. Sheikhzadeh, Catal. Commun., 2012, 19, 21

20. M. Hasanzadeh, N. Shadjou, E. Omidinia, M. Eskandani, M. de la Guardia, and P. Sheikhzadeh, Trends Anal. Chem., 2013, 45, 93 .

21. M. Hasanzadeh, N. Shadjou, and E. Omidinia, Colloids Surf., B, 2013, 108, 52.

22. M. Hasanzadeh, S. Sadeghi, L. Bageri, A. Mokhtarzadeh, N. Shadjou, and S. Mahboob, Mater. Sci. Eng. C, 2016, 69 , 343.

23. M. Hasanzadeh, A. Karimzadeh, N. Shadjou, A. Mokhtarzadeh, L. Bageri, S. Sadeghi, and S. Mahboob, Mater. Sci. Eng. C, 2016, 68, 814.

24. L. Saghatforoush, M. Hasanzadeh, N. Shadjou, and B. Khalilzadeh, Electrochim. Acta, 2011, 56, 1051.

25. L. Saghatforoush, M. Hasanzadeh, and N. Shadjou, Chin. Chem. Lett., 2014, 25, 655

26. N. Shadjou, M. Hasanzadeh, F. Talebi, and A. P. Marjani,

Nanocomposites, 2016, 2, 18.
27. E. Omidinia, N. Shadjou, and M. Hasanzadeh, Mater. Sci. 
Eng. C, 2013, 33, 4624.

28. E. Omidinia, N. Shadjou, and M. Hasanzadeh, Mater. Sci. Eng. C, 2014, 42, 368.

29. E. Omidinia, N. Shadjou, and M. Hasanzadeh, IEEE Sensors J., 2014, 14, 1081.

30. E. Omidinia, N. Shadjou, and M. Hasanzadeh, Appl. Biochem. Biotechnol., 2014, 172, 2070.

31. M. Hasanzadeh, N. Shadjou, A. Mokhtarzadeh, and M. Ramezani, Mater. Sci. Eng. C, 2016, 68, 482.

32. J. Soleymani, M. Hasanzadeh, N. Shadjou, M. K. Jafari, J. V. Gharamaleki, J. V. Gharamaleki, M. Yadollahie, and A. Jouyban, Mater. Sci. Eng. C, 2016, 61, 638.

33. L. A. Saghatforoush, M. Hasanzadeh, and N. Shadjou, Chin. Chem. Lett., 2014, 25, 655.

34. L. A. Saghatforoush, M. Hasanzadeh, and N. Shadjou, $J$. Electroanal. Chem., 2014, 714, 79.

35. M. Hasanzadeh, M. H. Pournaghi-Azar, N. Shadjou, and A. Jouyban, RSC Adv., 2014, 4, 6580.

36. M. Hasanzadeh and N. Shadjou, J. Nanosci. Nanotech., 2013, 13, 4909.

37. M. Hasanzadeh, N. Shadjou, and M. de la Guardia, Trends
Anal. Chem., 2015, 72, 123.

38. B. Khalilzadeh, N. Shadjou, H. Afsharan, M. Eskandani, H. N. Charoudeh, and M. R. Rashidi, BioImpacts, 2016, 6, 135.

39. M. Hasanzadeh, N. Hashemzadeh, N. Shadjou, J. EivaziZiaei, M. Khoubnasabjafari, and A. Jouyban., J. Mol. Liq., 2016, 221, 354.

40. M. Hasanzadeh, N. Shadjou, and M. Marandi, J. Nanosci. Nanotechnol., 2016, 17, 4598.

41. M. Hasanzadeh, F. Mokhtari, N. Shadjou, A. Eftekhari, A. Mokhtarzadeh, V. Jouyban-Gharamaleki, and S. Mahboob, Mater. Sci. Eng. C, 2017, 75, 247.

42. M. Hasanzadeh, N. Shadjou, and M. Marandi, Mater. Sci. Eng. C, 2017, 71, 1313.

43. M. Hasanzadeh, N. Shadjou, and M. Marandi, J. Alloys Compd., 2016, 688, 171.

44. J. Ou, Y. Tao, J. Xue, Y. Kong, J. Dai, and L. Deng, Electrochem. Commun., 2015, 57, 5.

45. A. J. Bard and L. R. Faulkner, "Electrochemical Methods: Fundamentals and Applications", 2nd ed., 2001, Wiley and Sons, Hoboken. 